www.nature.com/pj

\title{
Preparation methods of alginate micro-hydrogel particles and evaluation of their electrophoresis behavior for possible electronic paper ink application
}

\author{
Daisaku Kaneko, Nguyen Quyen Thi le, Tatsuya Shimoda and Tatsuo Kaneko
}

For the purpose of an electronic paper application, alginate/Ca hydrogel particles having a diameter of a few micrometers used with Titanium Black (TB), which has a diameter of $\sim 1 \mu \mathrm{m}$, for visualization purposes were prepared by mixing an aqueous sodium alginate solution with TB into an aqueous $\mathrm{CaCl}_{2}$ solution. In order to increase their absolute zeta potential ļ̧l for demonstrating good electric responsive behavior, micro-hydrogel particles were rinsed instantaneously by different concentrations of $\mathrm{Na}_{2} \mathrm{CO}_{3}$ solution. Through this procedure, the absolute value of the zeta potential $|\zeta|$ in pure water increased from $|\zeta|=1.3 \mathrm{mV}$ for the minimum value to $|\zeta|=73 \mathrm{mV}$ for the maximum value with an upon increasing $\mathrm{Na}_{2} \mathrm{CO}_{3}$ concentration. Micro-hydrogel particles were set between electrodes in pure water, their electro-responsive behavior was examined under an applied electric field and their position was analyzed using an optical microscopy. It was found that micro-hydrogel particles with a high absolute zeta potential $|\zeta|$ could oscillate from the anode to the cathode with a corresponding frequency, and threshold absolute zeta

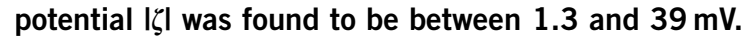

Polymer Journal (2010) 42, 829-833; doi:10.1038/pj.2010.78; published online 1 September 2010

Keywords: alginate hydrogel; electronic paper; electrophoresis; zeta potential

\section{INTRODUCTION}

It is well known that an aqueous solution of sodium alginate, a type of polysaccharide derived from seaweed that has a negatively charged polymer chain, forms hydrogel particles under the presence of multiply charged metal ions (for example, $\mathrm{Ca}^{2+}$ or $\mathrm{Mg}^{2+}$ ) due to the formation of ion crosslinking points. ${ }^{1,2}$ Recently, with the advancement of nanotechnology, alginate hydrogels are expected to be used in nano and/or micro devices because gelation can occur even at the nano and/ or micro scale range. Actually, using the inkjet printing technique, alginate hydrogels have been shaped into artificial capillary blood vessels. ${ }^{3}$ However, the collective position controlling of these independent micro-gel particles has not been realized because micro-gel particles are usually used in liquids and are highly dispersed when used in this form. Therefore, we have proposed a method of collective position controlling techniques based on the surface energy gradient or electrophoresis driving forces. ${ }^{4}$ The position of these micro-hydrogel particles could be controlled by electrophoresis on increasing their surface absolute zeta potential $|\zeta|$. To increase their surface absolute zeta potential $|\zeta|$, we examined the surface modification of microhydrogel particles by immersing them in an aqueous $\mathrm{Na}_{2} \mathrm{CO}_{3}$ and $\mathrm{HCl}$ solution for a few seconds. After this chemical modification, we confirmed that the absolute zeta potential $|\zeta|$ of micro-hydrogel particles increased from 1.3 to $73 \mathrm{mV}$ and the electro-responsive behavior of the micro-hydrogel particles drastically improved.
To the best of our knowledge, although there have been reports concerning the electrophoresis of micro-hydrogels, our method could be a more effective method than previously reported ones. ${ }^{5-7}$ In this paper, we will describe the details of our new procedures to modify the micro-hydrogel particles surfaces and report the analysis results of their electro-responsive behaviors. We believe that our results should significantly affect the electronic ink industry because alginate hydrogels do include various kinds of colored ink and can advance the contrast of electronic paper due to their softness.

\section{EXPERIMENTAL PROCEDURE}

Materials

The sodium alginate $(500-600 \mathrm{cp})$ was purchased from Wako, Osaka, Japan, and used as received. Calcium chloride, sodium carbonate and hydrochloric acid were purchased from NACALAI TESQUE (Kyoto, Japan), and used as received. A comb-type electrode was purchased from the CHISSO (Tokyo, Japan); details are described elsewhere. ${ }^{4}$ Titanium Black (TB, diameter $\sim 1 \mu \mathrm{m}$ ) was obtained from the Nippon Shokubai (Osaka, Japan).

\section{Preparation of micro-hydrogel particles}

Sodium alginate was dissolved in deionized water to make a $1.0 \mathrm{wt} \%$ solution and then a small amount of $\mathrm{Ti}$ or $\mathrm{TiO}_{2}$ for visualization purposes was mixed with the solution. This solution was dropped into a $0.03 \mathrm{M} \mathrm{CaCl}_{2}$ solution from a syringe to obtain micro-hydrogel particles. Because the resultant hydrogel particles widely vary in size, the hydrogel particles of a few microns in diameter 
were separated from the other particles by filter paper; their snapshot is shown in Figure 1.

\section{Surface modification of the negatively charged micro-hydrogel particle surfaces}

An aqueous $\mathrm{Na}_{2} \mathrm{CO}_{3}$ solution was prepared in various concentrations ranging from 0.01 to $0.05 \mathrm{M}$ in order to confirm concentration dependence for

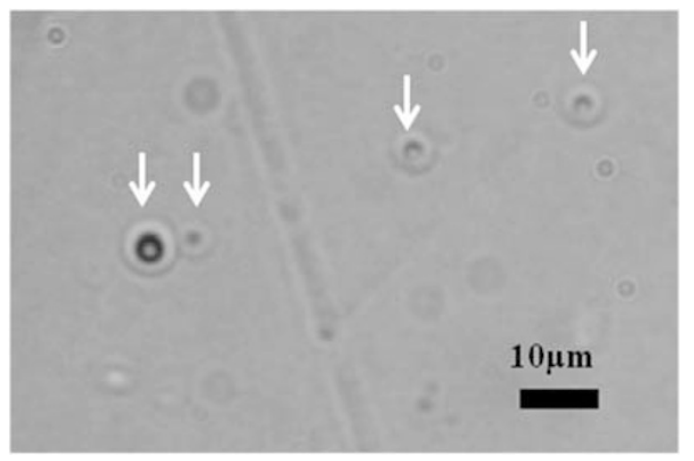

Figure 1 Snapshot of alginate micro-hydrogel particles with Titanium Black (TB) with $\sim 1-\mu \mathrm{m}$ diameter for well visualization. the improvement of the micro-hyrogel particles surfaces of absolute zeta potential $|\zeta|$.

In order to modify only the surface of the micro-hydrogel particles, microhydrogel particles were instantaneously rinsed by an aqueous $\mathrm{Na}_{2} \mathrm{CO}_{3}$ solution using a filtering apparatus with a vacuum pump. The pouring speed of the $\mathrm{Na}_{2} \mathrm{CO}_{3}$ solution $(50 \mathrm{ml})$ was $5 \mathrm{ml} \mathrm{s}^{-1}$. After this procedure, micro-hydrogel particles were washed with $50 \mathrm{ml} \mathrm{HCl}(0.12 \mathrm{~N})$. We then obtained surface modified micro-hydrogel particles after washing them with pure water five times. Through these successive procedures, the micro-hydrogel particles appeared to be soft. A schematic illustration of the procedure is shown in Figure 2.

\section{Measurements}

The zeta potential $\zeta$ of micro-hydrogel particles was measured in deionized water by the Zetasizer Nano (ZS-2000, Malvern Instruments, Worcestershire, UK), and the data collected are summarized in Figure 3b. Surface-modified or unmodified micro-hydrogel particles were dispersed in deionized water and placed between the electrodes with a distance of $10 \mu \mathrm{m}$ on the glass substrate. The electro-responsive behavior of the micro-hydrogel particles was examined by applying a voltage of $9.0 \mathrm{~V}$ (corresponding to an electric field of $E=9.0 \times 10^{5} \mathrm{~V} \mathrm{~m}^{-1}$ ) and $2.0 \mathrm{~Hz}$ using a digital function generator (DF1905) by an optical microscopy with a charge coupled device camera, recording movies with a frame rate of $30 \mathrm{fps}$.

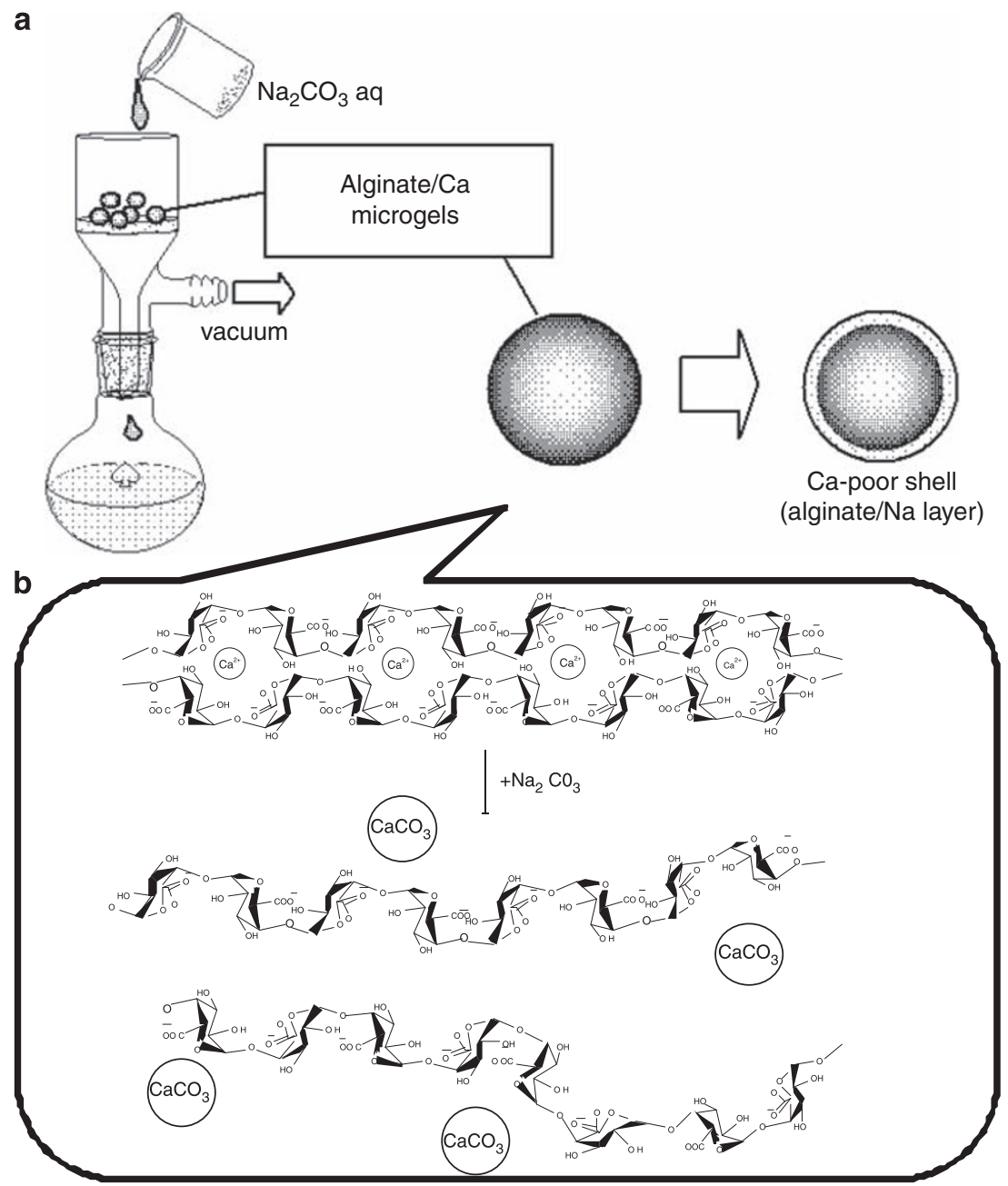

Figure 2 (a) Schematic illustration of the surface modification of micro-hydrogel particles. Micro-hydrogel particles were washed with an aqueous $\mathrm{Na}_{2} \mathrm{CO}_{3}$ solution for a few seconds in order to destroy surface ionic crosslinking points. After this simple procedure, the hydrogel surface seems to have a core-shell structure. (b) Schematic illustration of the fracture of ionic crosslinking points by treatment with aqueous $\mathrm{Na}_{2} \mathrm{CO}_{3}$ solution. Crosslinking points, named 'Egg-Box Junctions,' were destroyed, and alginate chains changed a grafted state. 


\section{RESULTS AND DISCUSSION}

Micro-hydrogel particles were made by dropping a $1 \mathrm{M}$ aqueous sodium alginate solution containing a small amount of Titanium Black (TB) ( $\sim 1 \mu \mathrm{m}$ in diameter) from a syringe into an aqueous 0 . $03 \mathrm{M} \mathrm{CaCl}_{2}$ solution for visualization purposes. Because the hydrogel particle size varies throughout a wide range, hydrogel particles a few microns in diameter were separated by filter paper. Figure 1 shows a snapshot of the separated hydrogel particles in water. Details of the micro-hydrogel particle preparation are described in the experimental procedure section.

At this stage, we expect that if these micro-hydrogel particles in water have negatively charged surfaces due to their negatively charged polymer chains, these micro-hydrogel particles should show electroresponsive (electrophoresis) behavior under an electric field. However, electro-responsive behavior could not be observed at all. We concluded that no response resulted from the low zeta potential of the micro-hydrogel particles. We then examined the measurement of the zeta potential $\zeta$ of these micro-hydrogel particles, which was found to be only $-1.3 \mathrm{mV}$. The zeta potential $\zeta$ of micro-hydrogel particles was determined by the Helmholtz-Smoluchowski equation,

$$
\zeta=\frac{\eta}{\varepsilon} \cdot \frac{v}{V}
$$

where $\eta$ and $\varepsilon$ are the viscosity and the dielectric constant of the fluid, respectively. $V$ is the applied voltage, and $v$ is the relative velocity of the micro-hydrogel particles. A low zeta potential may result from the

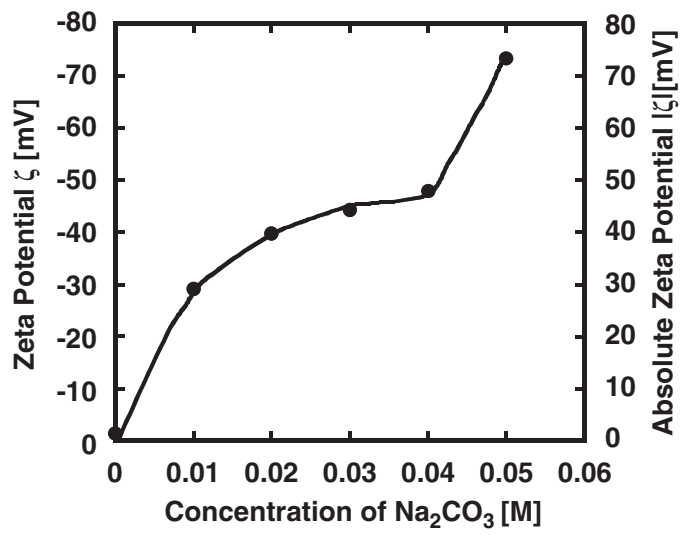

Figure 3 Change in zeta potential, $\zeta$, as a function of the $\mathrm{Na}_{2} \mathrm{CO}_{3}$ concentration. electric cancellation of the negatively charged polymer chain with the presence of the excessive $\mathrm{Ca}^{2+}$ ions. Therefore, to remove these excessive $\mathrm{Ca}^{2+}$ ions, the micro-hydrogel particles were immersed in an aqueous $\mathrm{Na}_{2} \mathrm{CO}_{3}$ solution, as shown in Figure 2a, because the $\mathrm{CO}_{3}{ }^{2-}$ ion strongly traps $\mathrm{Ca}^{2+}$ ions. Moreover, we also expected the $\mathrm{CO}_{3}{ }^{2-}$ ion destroys the crosslinking points of the micro-hydrogel surface by trapping the $\mathrm{Ca}^{2+}$ ions that form crosslinking points. A schematic image of the fracture of the crosslinking points is shown in Figure 2b. From the microscopy, we observed that the microhydrogel particles seem to have a core-shell structure (data not shown). Details of this procedure (the chemical surface modification of micro-hydrogel particles) are described in the experimental procedure section.

It was found that the absolute zeta potential $|\zeta|$ of surface-modified micro-hydrogel particles increases with an increase in the concentration of the aqueous $\mathrm{Na}_{2} \mathrm{CO}_{3}$ solution, as shown in Figure 3. The maximum value of the absolute zeta potential $|\zeta|$ was found to be $73 \mathrm{mV}$ after treatment with an aqueous $0.05 \mathrm{M} \mathrm{Na}_{2} \mathrm{CO}_{3}$ solution. The $73 \mathrm{mV}$ value for the absolute zeta potential is reported to be the nearly maximum value for the following two reasons: (1) the absolute zeta potential $|\zeta|$ of an aqueous $1 \mathrm{~m}$ alginate solution (which was not crosslinked and was the same concentration of preparation solution for the micro gels; see the experimental procedure section) is nearly $90 \mathrm{mV}$ and (2) using a larger concentration of $\mathrm{Na}_{2} \mathrm{CO}_{3}$ in aqueous solution than $0.05 \mathrm{M}$ destroyed the micro-hydrogel particles.

We then examined the stability of the dispersibility in pure water. For comparison, (a) TB, (b) unmodified micro-hydrogel particles and (c) surface-modified micro-hydrogel particles were each dispersed into pure water and observed at 0 and $48 \mathrm{~h}$ after dispersion. Figure 4 shows the ultraviolet absorbance spectrum. Even in the initial state $(0 \mathrm{~h})$, the dispersion of the unmodified micro-hydrogel particles (b) was inferior to that of TB (a) and surface-modified micro-hydrogel particles (c). After $48 \mathrm{~h}$, the surface-modified particles (c) kept a higher dispersion level than that of TB (a) and unmodified particles (b). These results suggest that the surface-modified micro-hydrogel particles were an improvement over $\mathrm{TB}$ and unmodified particles in their temporal stability due to their repulsions due to their negative charge.

Using these surface-modified micro-hydrogel particles, we examined their electro-responsive behavior under an applied electric field of $E_{\mathrm{c}}=9.0 \times 10^{5} \mathrm{~V} \mathrm{~m}^{-1}$ (corresponding voltage is $9.0 \mathrm{~V}$ and frequency is 1.0 and $2.0 \mathrm{~Hz}$ ). Surface-modified micro-hydrogel particles were dispersed in deionized water and placed between electrodes. Details of the electrodes are described elsewhere. ${ }^{4}$ Figure 5 shows snapshots of the electro-responsive behavior of surface-modified micro-hydrogel

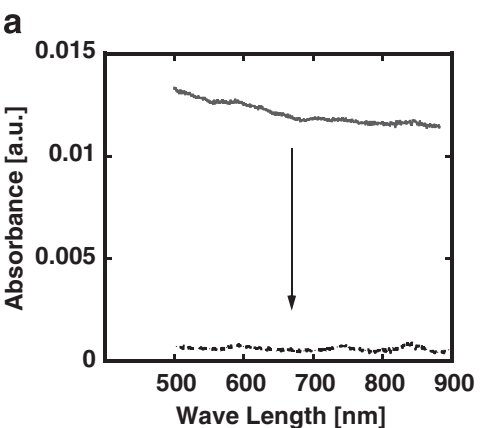

b

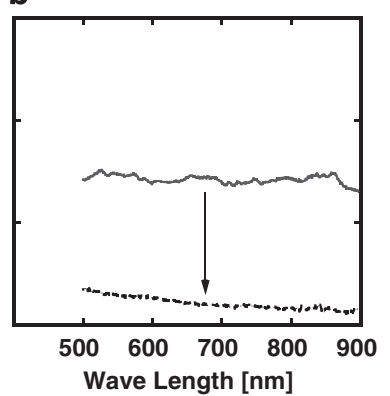

C

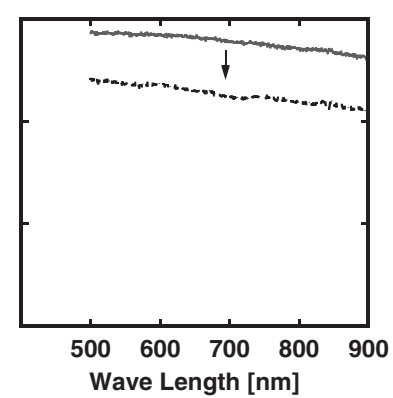

Figure 4 Ultraviolet absorbance spectrum of (a) Titanium Black (TB), (b) alginate micro-hydrogel particles with TB and (c) surface-modified alginate microhydrogel particles with TB dispersed in pure water. Continuous lines and the dotted line in the graphs represent the data taken between 0 and $48 \mathrm{~h}$ after the dispersion. 

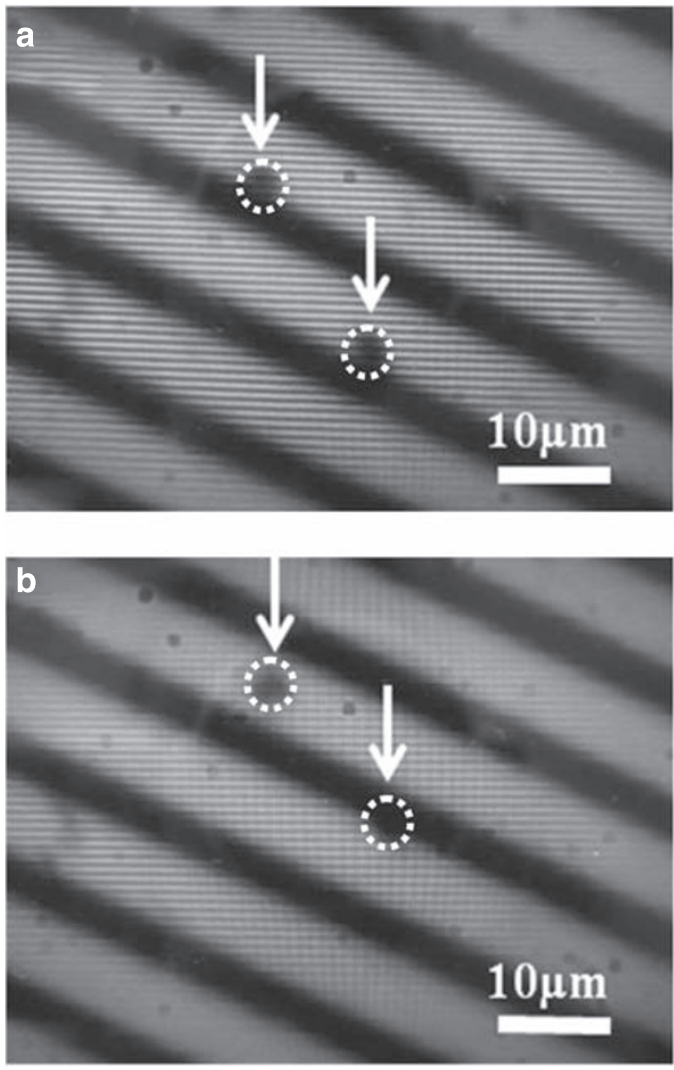

Figure 5 Snapshot of the electro-responsive behavior of alginate/Titanium Black (TB) composite particles under an applied AC voltage of $9 \mathrm{~V}$ at $2 \mathrm{~Hz}$. Parallel black lines are alternately located cathode and anode electrodes. The absolute zeta potential $|\zeta|$ is $41 \mathrm{mV}$. (a) $0 \mathrm{~s}$ and (b) $2 \mathrm{~s}$ after starting the measurement. Dotted circles in the pictures indicate the hydrogel particles are $\sim 3 \mu \mathrm{m}$ in diameter.

particles having an absolute zeta potential of $|\zeta|=41 \mathrm{mV}$ under an applied voltage of $9.0 \mathrm{~V}$ and a frequency of $2.0 \mathrm{~Hz}$. The arrows and dotted circles in the figure indicate the micro-hydrogel particles. It was confirmed that the micro-hydrogel particles were oscillating according to the applied electric field $E_{\mathrm{c}}$. The position analysis in the case of absolute zeta potential $|\zeta|=1.3$ and $41 \mathrm{mV}$ was also examined using the same conditions and plotted in Figure 6. It was found that the position $Y$ of micro-hydrogel particles could be fitted well by sine curves as follows:

$$
Y=A_{0} \sin 2 \pi f\left(t-t_{0}\right)
$$

where $A_{0}$ and $f$ represent the vibration amplitude and frequency, respectively, and $t$ is the measuring time. The value of $t_{0}$ has a dimension of time and is used to fit the sine curve from obtained data. Each parameter is summarized in Table 1. From Figure 6, in the case of $|\zeta|=1.3 \mathrm{mV}$, electro-responsive behavior could not be observed at all. On the other hand, in the case of the high absolute value of the zeta potential $|\zeta|=41 \mathrm{mV}$, a micro-hydrogel particle can oscillate at 0.88 and $2.00 \mathrm{~Hz}$ when a frequency of 1.0 and $2.0 \mathrm{~Hz}$ is applied, respectively (the voltage is $9.0 \mathrm{~V}$ ). From Table 1 , the vibration amplitude $A_{0}$ in the case of $1.0 \mathrm{~Hz}$ appears larger than that in the $2.0 \mathrm{~Hz}$ case. This phenomenon is because the $1.0 \mathrm{~Hz}$ case produces enough time for micro-hydrogel particles to move. We confirmed that the threshold absolute zeta potential $|\zeta|$ exists between 1.3 and $29 \mathrm{mV}$, and the maximum response frequency is confirmed $\sim 4 \mathrm{~Hz}$ in the case of the 73-mV absolute zeta potential (data not shown). These results

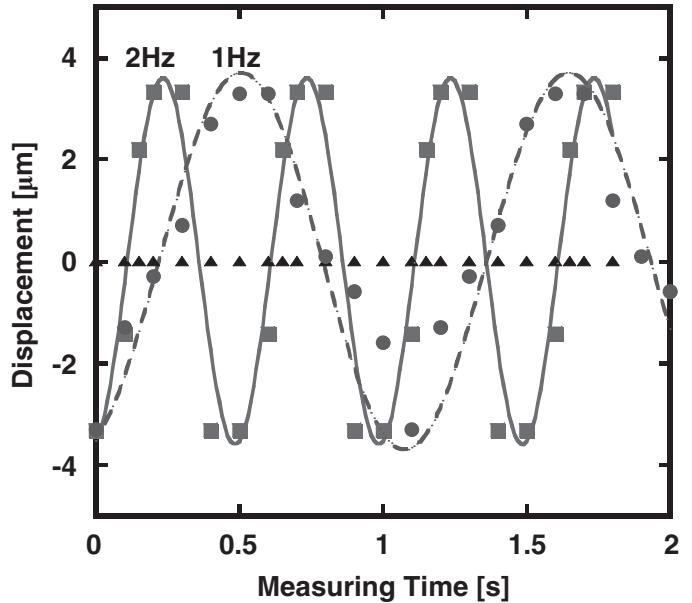

Figure 6 Displacement of micro-hydrogel particles under $9 \mathrm{~V}$ applied voltage. Filled triangles $(\boldsymbol{\Delta})$ denote the case of the $-1.3-\mathrm{mV}$ zeta potential. Filled squares $(\boldsymbol{\square})$ and circles $(\bullet)$ denote the case of the $-41-\mathrm{mV}$ zeta potential under 1 and $2 \mathrm{~Hz}$, respectively, and with the applied voltage of $9 \mathrm{~V}$. Continuous lines and dotted lines in the figure are curve fits from Equation (2) for the cases of 1 and $2 \mathrm{~Hz}$, respectively. The distance between electrodes is $10 \mu \mathrm{m}$, and a displacement of zero denotes the center position between electrodes.

Table 1 Parameters of Equation (2)

\begin{tabular}{lcccc}
\hline$|\zeta|(m V)$ (Frequency) & $A_{0}(\mu \mathrm{m})$ & $f(\mathrm{~Hz})$ & $t_{0}(\mathrm{~s})$ & $\mathrm{R}^{2}$ \\
\hline $1.3(1 \mathrm{~Hz})$ & 0 & - & - & - \\
$41(1 \mathrm{~Hz})$ & 3.7 & $0.88 \pm 0.02$ & $0.220 \pm 0.020$ & 0.85 \\
$41(2 \mathrm{~Hz})$ & 3.6 & $2.00 \pm 0.02$ & $0.610 \pm 0.006$ & 0.95 \\
\hline
\end{tabular}

indicate that the micro-hydrogel particles having a higher absolute zeta potential $|\zeta|$ are closer to the applied electric frequency.

Although our methods and findings still remain at a phenomenological level at this stage, our proposed methods to give an electrophoresis property effectively for the micro-hydrogel particles has a tremendous amount of potential for the next-generation electronic paper ink industry. These hydrogels can fulfill the demands of the next generation of electronic paper ink by their high electro-responsiveness, high contrast and high adhesive properties as a transparent substrate.

\section{Summary}

For the application of the next generation of electronic paper ink, a manner in which to control the position of alginate micro-hydrogel particles dispersed in liquid has been proposed. We have investigated a very simple method to drastically improve the zeta potential, $\zeta$, of alginate micro-hydrogel particles for electrophoresis behavior by incubating them with an aqueous $\mathrm{Na}_{2} \mathrm{CO}_{3}$ solution for a few seconds. To the best of our knowledge, the chemical modification method described in this paper is the simplest and first report to improve the zeta potential in this manner. The electro-responsive behaviors of micro-hydrogel particles were also examined using modified and unmodified micro-hydrogel particles under an electric field. It was found that with an increase in the absolute zeta potential $|\zeta|$ of the micro-hydrogel particles, the electro-responsive behavior of the particles became sensitive to the applied frequency, and the 
surface-modified hydrogel particles had a high enough response ability for application as the next-generation of electronic paper ink.

1 Rendevski, S. J. \& Andonovoski, A. N. Microgel structures calcium alginate in aqueous solutions Proceding Suplement of BPU-5, SP06-103, August 25-29, Vrnjacka Banja, SMN. 161-165 (2003).

2 lijima, M., Hatakeyama, T., Nakamura, K. \& Hatakeyama, H. Thermomechanical analysis of calcium alginate hydrogels in water. J Thermal Anal Calorimetry 70, 807-814 (2002). 3 Henmi, C., Nakamura, M., Nishiyama, Y., Yamaguchi, K., Mochizuki, S., Takiura, K. \& Nakagawa, H. Development of an effective three dimensional fabrication technique using inkjet technology for tissue model samples. Proceedings 6th World Congress on Alternatives \& Animal Use in the Life Sciences 14, 689-692 (2007).

4 Kaneko, D., Shibata, K., Kaneko, T. \& Kawakami, Y. Transportation of a microdroplet on an oriented liquid crystal surface. Liquid Crystals 35, 661-664 (2008).

5 Park, J. H., Lee, M. A., Park, B. J. \& Choi, H. J. Preparation and electrophoretic response of poly(methyl methacrylate-co-methacrylic acid) coated $\mathrm{TiO}_{2}$ nanoparticles for electronic paper application. Curr Appl Phys 7, 349-351 (2007).

6 Fang, X., Yang, H., Wu, G., Li, W., Chen, H. \& Wang, M. Preparation and characterization of low density polystyrene/ $/ \mathrm{TiO}_{2}$ core-shell particles for electronic paper application. Curr Appl Phys 9, 755-759 (2009).

7 Park, S. L., Park, J. W., Choi, H. Y., Han, Y. S., Kwon, Y. \& Choi, H. S. Fabrication of charged particles for electrophoretic display. Curr Appl Phys 6, 644-648 (2006). 